\title{
Reversible Simulation of Bipartite Product Hamiltonians
}

\author{
Andrew M. Childs, Debbie W. Leung, and Guifré Vidal
}

\begin{abstract}
Consider two quantum systems $A$ and $B$ interacting according to a product Hamiltonian $H=H_{A} \otimes H_{B}$. We show that any two such Hamiltonians can be used to simulate each other reversibly (i.e., without efficiency losses) with the help of local unitary operations and local ancillas. Accordingly, all nonlocal features of a product Hamiltonian-including the rate at which it can be used to produce entanglement, transmit classical or quantum information, or simulate other Hamiltonians-depend only upon a single parameter. We identify this parameter and use it to obtain an explicit expression for the entanglement capacity of all product Hamiltonians. Finally, we show how the notion of simulation leads to a natural formulation of measures of the strength of a nonlocal Hamiltonian.
\end{abstract}

Index Terms-Channel capacity, entanglement, Hamiltonian simulation, quantum control, quantum information theory.

\section{INTRODUCTION}

$\mathbf{S}$ UPPOSE two quantum systems $A$ and $B$ are coupled by some nontrivial interaction Hamiltonian $H \neq H_{A} \otimes I_{B}+$ $I_{A} \otimes H_{B}$. Such a Hamiltonian can be used for a variety of information-processing tasks, such as transmitting classical or quantum information, creating entanglement, or simulating other nonlocal evolutions. One of the goals of quantum information theory is to quantify the capacity of an interaction to perform such information-processing tasks.

The various nonlocal properties of Hamiltonians can be analyzed in different ways, but they are typically studied under a common framework of perfect local control. To focus on the uniquely nonlocal features of Hamiltonians, all local abilities are regarded as free resources. This includes the use of local ancillary degrees of freedom and the ability to perform fast local operations to modify the evolution.

One of the tasks mentioned above consists of using $H$ and local operations to simulate another Hamiltonian $H^{\prime}$, a control technique that allows one to modify a naturally available interaction into a more convenient one. Any bipartite interac-

Manuscript received March 27, 2003; revised July 22, 2003. The work of A. M. Childs was supported by the Fannie and John Hertz Foundation, and, in part, by the Cambridge-MIT Foundation, the Department of Energy under cooperative research agreement DE-FC02-94ER40818, and the National Security Agency and Advanced Research and Development Activity under Army Research Office Contract DAAD19-01-1-0656. The work of D. W. Leung and G. Vidal was supported by the National Science Foundation under Grant EIA0086038 .

A. M. Childs is with the Center for Theoretical Physics, Massachusetts Institute of Technology, Cambridge, MA 02139 USA (e-mail: amchilds@ @it.edu).

D. W. Leung and G. Vidal are with the Institute for Quantum Information, California Institute of Technology, Pasadena, CA 91125 USA (e-mail: wcleung@cs.caltech.edu; vidal@cs.caltech.edu).

Communicated by E. H. Knill, Associate Editor for Quantum Information Theory.

Digital Object Identifier 10.1109/TIT.2004.828069 tion Hamiltonian can simulate any other at some nonzero rate [1]-[4]. However, we would ultimately like to know the most efficient way to use $H$ to simulate $H^{\prime}$, as well as the optimal rate $\gamma_{H^{\prime} \mid H}$ at which the simulation can be accomplished. A method for optimal simulation of two-qubit Hamiltonians is given in [2], and the optimal rate in this case can be expressed in terms of a majorization condition [5]. However, to the best of our knowledge, no optimal simulation rates have been reported beyond the two-qubit case.

Understanding Hamiltonian simulation also provides insight into capacities for other information-processing tasks. Let $C_{H}$ denote the capacity of Hamiltonian $H$ to accomplish one of these tasks, again assuming perfect local control. If Hamiltonian $H$ can be used to simulate $H^{\prime}$ at a rate $\gamma_{H^{\prime} \mid H}$, then

$$
C_{H} \geq \gamma_{H^{\prime} \mid H} C_{H^{\prime}}
$$

since one could first use $H$ to simulate $H^{\prime}$ and then use $H^{\prime}$ to accomplish the task. Equation (1) is a lower bound on the capacity of $H$, or equivalently, an upper bound on the capacity of $H^{\prime}$. Of course, such bounds need not be tight. For example, the majorization condition for optimal simulation of two-qubit Hamiltonians only provides a partial order on these Hamiltonians, and thus the resulting bounds on capacities-for example, on the entanglement capacity [6]-[8]—are not always tight.

The fact that any nonlocal Hamiltonian can simulate any other at some nonzero rate means that all interactions are qualitatively equivalent. A much stronger, quantitative notion of equivalence between interactions comes from the possibility of performing a reversible simulation. We say that $H$ and $H^{\prime}$ can simulate each other reversibly if we can use $H$ to simulate $H^{\prime}$, and then use $H^{\prime}$ to simulate $H$ back, with no overall loss in efficiency. In terms of simulation rates, reversible simulation amounts to the condition

$$
\gamma_{H \mid H^{\prime}} \gamma_{H^{\prime} \mid H}=1
$$

Notice that if two Hamiltonians $H$ and $H^{\prime}$ can simulate each other reversibly, then their capacities are related by

$$
C_{H}=\gamma_{H^{\prime} \mid H} C_{H^{\prime}}
$$

as can be seen by applying (1) in both directions. Furthermore, if every pair of Hamiltonians in some given set can simulate each other reversibly, then simulation provides a total order on the set. Thus, the nonlocal properties of the entire set can be studied by focusing on only one Hamiltonian in the set.

In this paper, we consider the set of bipartite Hamiltonians that can be written as a tensor product of the form

$$
H=H_{A} \otimes H_{B}
$$


where $H_{A}$ acts on system $A$ and $H_{B}$ acts on system $B$. We shall call such a Hamiltonian a product Hamiltonian for short. An example of a product Hamiltonian in a two-qubit system is the Ising interaction $H_{\text {Ising }}$

$$
H_{\text {Ising }}:=\sigma_{z} \otimes \sigma_{z}, \quad \sigma_{z}:=\left[\begin{array}{cc}
1 & 0 \\
0 & -1
\end{array}\right] .
$$

Our main result is an explicit protocol for the reversible simulation of any product Hamiltonian by another. It follows that the nonlocal properties of a product Hamiltonian $H$ depend entirely on a single parameter. We denote this parameter by $K_{\otimes}(H)$, and choose it to be the rate $\gamma_{H_{\text {Ising }} \mid H}$ at which $H$ can simulate the Ising interaction. We find that

$$
K_{\otimes}(H)=\frac{1}{4} \Delta_{A} \Delta_{B}
$$

where $\Delta_{A}\left(\Delta_{B}\right)$ denotes the difference between the largest and the smallest eigenvalues of $H_{A}\left(H_{B}\right)$. The optimal simulation rate between any two product Hamiltonians $H$ and $H^{\prime}$ can be written in terms of $K_{\otimes}$ as

$$
\gamma_{H^{\prime} \mid H}=\frac{K_{\otimes}(H)}{K_{\otimes}\left(H^{\prime}\right)}
$$

so that any capacity known for just one product Hamiltonian can be easily computed for any other product Hamiltonian using (3) and (7). In particular, we use previous results for the Ising interaction [8] to obtain a simple expression for the entanglement capacity of any product Hamiltonian.

In addition to quantifying the ability of an interaction to perform particular tasks, one can imagine defining abstract measures of the nonlocality of an interaction. Reference [9] introduced the notion of a strength measure $K(U)$ of a nonlocal quantum operation. Three axioms that every strength measure should satisfy were proposed, along with several additional desirable properties. Here we consider strength measures $K(H)$ for nonlocal Hamiltonians. We will formulate a single axiom in terms of Hamiltonian simulation that implies many desirable properties. For the special case of product Hamiltonians, our results imply an essentially unique measure, $K_{\otimes}(H)$ in (6), with several additional properties.

The structure of the paper is as follows. In Section II, we describe the problem of bipartite Hamiltonian simulation in more detail and discuss a set of basic simulation rules that can be used to build up all possible simulations by composition. In Section III, we derive our main result, namely, that all tensor product Hamiltonians can reversibly simulate each other. In Section IV, we apply this result to the calculation of entanglement capacities, and in Section V, we relate Hamiltonian simulation to the strength measure formalism. Finally, in Section VI, we conclude with a discussion of our findings and of some open problems.

\section{Simulating BipARTite HAMiltonians}

The problem of bipartite Hamiltonian simulation can be posed as follows. We consider two quantum systems $A$ and $B$ held by Alice and Bob, respectively. The systems interact according to some nonlocal Hamiltonian $H \neq H_{A} \otimes I_{B}+$ $I_{A} \otimes H_{B}$. Alice and Bob want to use $H$ to produce an evolution according to some other bipartite Hamiltonian $H^{\prime}$. In order to do so, they are given the additional resources of synchronized clocks and perfect local control. They may attach or discard local ancillary systems and they may apply (arbitrarily fast) local operations, which can be assumed to be unitary without loss of generality [2]. As a side remark, classical communication between Alice and Bob would not increase the optimal simulation rate [5]. Note that the goal of the simulation is not to produce the Hamiltonian evolution $e^{-i H^{\prime} t}$ for a particular time $t$, but rather to stroboscopically track the evolution $e^{-i H^{\prime} t}$ for arbitrarily closely spaced values of time. A detailed formulation of the problem can be found in [2].

We next present a list of rules for nonlocal Hamiltonian simulation. By composition, these rules give rise to all possible simulations achievable with local operations and ancillary systems. We present five basic rules, as well as three additional rules that can be obtained by combining the basic ones. We use the shorthand notation $H \longrightarrow H^{\prime}$ to represent the possibility of simulating $H^{\prime}$ by $H$ at the rate $\gamma_{H^{\prime} \mid H}$, and the notation $H \longleftrightarrow H^{\prime}$ to indicate that, in addition, the simulation can be reversed without overall efficiency losses, as in (2). We say that two Hamiltonians are locally equivalent if they can simulate each other reversibly at unit rate.

The first two basic rules merely make precise the notion of Hamiltonian evolution. They do not involve any operational procedure, nor assume any ability to control the system. The first rule makes precise the notion of rescaling the evolution time: a Hamiltonian $H$ can reversibly simulate another Hamiltonian $H^{\prime}=c H$ that only differs by a positive multiplicative constant $c$.

Rule 1-Rescaling: For any $c>0$

$$
H \longleftrightarrow c H, \quad \gamma_{c H \mid H}=\frac{1}{c} .
$$

Note that it is important that $c>0$. In general, Hamiltonians $H$ and $-H$ cannot simulate each other reversibly (see [2], [3] for examples).

The second rule makes precise what it means for a Hamiltonian to act on a subsystem. In the bipartite setting, the complete system can be described by subsystems $A, B$ on which $H$ acts and ancillary subsystems $A^{\prime}, B^{\prime}$ on which it acts trivially.

Rule 2-Ancillas: For any dimension of the ancillary Hilbert space $\mathcal{H}_{A^{\prime}} \otimes \mathcal{H}_{B^{\prime}}$

$$
H \longleftrightarrow H \otimes I_{A^{\prime} B^{\prime}}, \quad \gamma_{H \otimes I_{A^{\prime} B^{\prime}} \mid H}=1 .
$$

The next two basic rules arise from the possibility of switching on local Hamiltonians.

Rule 3-Local Hamiltonians: Any local Hamiltonian of the form $H_{0}=H_{A} \otimes I_{B}+I_{A} \otimes H_{B}$ can be produced at no cost. Recall that for any unitary transformation $U$, we have

$$
U e^{-i H t} U^{\dagger}=e^{-i U H U^{\dagger} t}
$$

Therefore, by means of local unitaries, any Hamiltonian $H$ is locally equivalent to any other that is obtained from it by local unitary conjugation. 
Rule 4-Local Unitaries: For any local unitary operation $U=U_{A} \otimes U_{B}$

$$
H \longleftrightarrow U H U^{\dagger}, \quad \gamma_{U H U^{\dagger} \mid H}=1 .
$$

Rules 1-4 allow Alice and Bob to produce Hamiltonians that differ from the original interaction $H$. The Lie-Trotter product formula

$$
e^{-i\left(H_{1}+H_{2}\right) t}=\lim _{n \rightarrow \infty}\left(e^{-i H_{1} t / n} e^{-i H_{2} t / n}\right)^{n}
$$

tells us how two of these Hamiltonians can be combined into a new one by alternately simulating each of them individually. With the help of Rule 1 we obtain the last basic rule.

Rule 5-Convex Combination: For any $H_{1}, H_{2}$, and $0 \leq$ $p \leq 1$, the simulation

$$
\left.\begin{array}{c}
p H_{1} \\
(1-p) H_{2}
\end{array}\right\} \longrightarrow H^{\prime}=p H_{1}+(1-p) H_{2}
$$

is possible with rate $\gamma_{H^{\prime} \mid p H_{1} ;(1-p) H_{2}} \geq 1$.

Here we have considered the use of Hamiltonian $H_{1}$ for a fraction of time $p$ and Hamiltonian $\mathrm{H}_{2}$ for a fraction of time $1-p$, and the rate of simulating $H^{\prime}$ is computed by adding these two times together. Let us stress that (13) assumes only the local ability to switch on and off the constituent Hamiltonians, and that only one Hamiltonian is acting at a time. Notice that this is the only basic simulation rule where irreversibility may occur. Although Alice and Bob can use $H^{\prime}$ to simulate back $H_{1}$ and $H_{2}$, in general they will incur an overall loss in efficiency by doing so.

These basic rules can be combined in various ways. We state three particularly useful combinations as additional rules. First, from Rules 3 and 5, a local part $H_{0}$ can be added to the given nonlocal Hamiltonian reversibly.

\section{Rule 6-Adding a Local Hamiltonian:}

$$
H \longleftrightarrow H+H_{0}, \quad \gamma_{H+H_{0} \mid H}=1 .
$$

Second, local unitary conjugation and convex combination can be composed into what we shall call a local unitary mixing of $H$.

Rule 7-Local Unitary Mixing: For any set of local unitary transformations $U_{i}=U_{A, i} \otimes U_{B, i}$ and any probability distribution $p_{i}\left(p_{i} \geq 0\right.$ and $\left.\sum_{i} p_{i}=1\right)$

$$
H \longrightarrow H^{\prime}=\sum_{i} p_{i} U_{i} H U_{i}^{\dagger}, \quad \gamma_{H^{\prime} \mid H} \geq 1 .
$$

Note that Rules 3-7 are stated without assuming local control over the ancillas. In the two-qubit case, Rules 1, 6, and 7 describe all relevant simulations because local control over ancillas is known to be unnecessary for optimal simulations [2].

More generally, we allow local control over ancillas in our simulation model. By Rule 2, Rules 3-7 can be extended to include ancillas as well. Control over ancillas gives extra freedom in the simulation, and is known to improve the achievable simulation rates in some cases [5].

Our last rule is concerned with any simulation in which the original Hamiltonian $H$ and the simulated Hamiltonian $H^{\prime}$ act on systems with different dimensions. Let $\mathcal{H}_{A} \otimes \mathcal{H}_{B}$ and $\mathcal{H}_{A^{\prime}} \otimes$ $\mathcal{H}_{B^{\prime}}$ denote the Hilbert spaces on which $H$ and $H^{\prime}$ act, with dimensions $d_{A}, d_{B}$ and $d_{A^{\prime}}, d_{B^{\prime}}$, where $d_{A} \geq d_{A^{\prime}}$ and $d_{B} \geq d_{B^{\prime}}$. For simplicity, we assume that $H=H_{A} \otimes H_{B}$ is a product Hamiltonian. If it were not, then we could expand $H$ as a linear combination of product Hamiltonians, $H=\sum_{i} H_{A, i} \otimes H_{B, i}$, and the following would hold for each of the terms in the expansion. Let vectors $|j\rangle_{A}\left(1 \leq j \leq d_{A}\right)$ denote an orthonormal basis in $\mathcal{H}_{A}$. We can express $H_{A}$ as

$$
H_{A}=\left[\begin{array}{ll}
J_{\|} & C^{\dagger} \\
C & J_{\perp}
\end{array}\right]
$$

where $J_{\|}$is the restriction of $H_{A}$ onto the subspace $\mathcal{H}_{A \|} \subseteq \mathcal{H}_{A}$ spanned by vectors $|j\rangle_{A}\left(1 \leq j \leq d_{A^{\prime}}\right)$, and $J_{\perp}$ the restriction onto its orthogonal complement. Consider also an analogous decomposition for $H_{B}$. Then we have the following.

Rule 8-Reduction to a Local Subspace: The simulation

$$
H=\left[\begin{array}{cc}
J_{\|} & C^{\dagger} \\
C & J_{\perp}
\end{array}\right] \otimes\left[\begin{array}{cc}
K_{\|} & D^{\dagger} \\
D & K_{\perp}
\end{array}\right] \longrightarrow H^{\prime}=J_{\|} \otimes K_{\|}
$$

is possible with rate $\gamma_{H^{\prime} \mid H} \geq 1$.

The last rule can be obtained by using the following lemma twice. The lemma shows how to simulate $J_{\|} \otimes H_{B}$ using $H_{A} \otimes$ $H_{B}$.

Lemma 1: The simulation

$$
H=\left[\begin{array}{cc}
J_{\|} & C^{\dagger} \\
C & J_{\perp}
\end{array}\right] \otimes H_{B} \longrightarrow H^{\prime}=J_{\|} \otimes H_{B}
$$

is possible with rate $\gamma_{H^{\prime} \mid H} \geq 1$.

Proof: We divide the simulation into two steps. i) First, by unitary mixing (Rule 7) with

$$
\begin{array}{ll}
p_{1}=\frac{1}{2}, & U_{1}=I_{A} \otimes I_{B} \\
p_{2}=\frac{1}{2}, & U_{2}=\left[\begin{array}{cc}
I_{\|} & 0 \\
0 & -I_{\perp}
\end{array}\right] \otimes I_{B}
\end{array}
$$

where $I_{\|}$and $I_{\perp}$ denote restrictions of the identity operator, we achieve the simulation

$$
H=\left[\begin{array}{cc}
J_{\|} & C^{\dagger} \\
C & J_{\perp}
\end{array}\right] \otimes H_{B} \longrightarrow H^{\prime \prime}=\left[\begin{array}{cc}
J_{\|} & 0 \\
0 & J_{\perp}
\end{array}\right] \otimes H_{B}
$$

with unit rate, so $\gamma_{H^{\prime \prime} \mid H} \geq 1$. ii) Second, we use $H^{\prime \prime}$ to simulate $H^{\prime}=J_{\|} \otimes H_{B}$ as follows. Suppose the goal is to evolve $|\psi\rangle_{A^{\prime} B}$ according to $e^{-i H^{\prime} t}$. We assume system $A$ is in state $|1\rangle_{A}$ by local control. Therefore, the joint state of systems $A A^{\prime} B$ is initially $|1\rangle_{A}|\psi\rangle_{A^{\prime} B}$. Let $V_{A A^{\prime}}$ denote a unitary transformation such that

$$
V_{A A^{\prime}}|1\rangle_{A}|j\rangle_{A^{\prime}}=|j\rangle_{A}|1\rangle_{A^{\prime}}, \quad 1 \leq j \leq d_{A^{\prime}} .
$$

Then the following three steps can be used to complete the desired simulation.

1) We apply unitary $V_{A A^{\prime}}$, placing $|\psi\rangle_{A^{\prime} B}$ in the subspace $\mathcal{H}_{A \|} \otimes \mathcal{H}_{B} \subset \mathcal{H}_{A} \otimes \mathcal{H}_{B}$

2) We make $A B$ evolve according to $H^{\prime \prime}$. Notice that at all times $e^{-i H^{\prime \prime} t}|\psi\rangle_{A B}$ is supported in $\mathcal{H}_{A \|} \otimes \mathcal{H}_{B}$, and that $H^{\prime \prime}$ acts on this subspace as $J_{\|} \otimes H_{B}$. 
3) We reverse $V_{A A^{\prime}}$, so that the net evolution on $A^{\prime} B$ has been $e^{-i H^{\prime} t}|\psi\rangle_{A^{\prime} B}$.

This completes the proof.

\section{REVERSIBLE SIMULATION}

In this section, we present the main result of the paper, the reversible simulation of tensor product Hamiltonians $H=H_{A} \otimes$ $H_{B}$. We will consider product Hamiltonians in a certain standard form. Using Rule 4, we may diagonalize $H_{A}$ and $H_{B}$, so we need only consider their eigenvalues. It will also be convenient to modify $H_{A}$ so that the largest and smallest eigenvalues $\lambda_{A}^{\max }$ and $\lambda_{A}^{\min }$ are equal in magnitude, and similarly for $H_{B}$. This can be done by adding a term proportional to the identity to each of $H_{A}$ and $H_{B}$, i.e.,

$$
\begin{aligned}
& \left(H_{A}+c I\right) \otimes\left(H_{B}+d I\right) \\
& \quad=H_{A} \otimes H_{B}+c I \otimes H_{B}+d H_{A} \otimes I+c d I \otimes I .
\end{aligned}
$$

The resulting Hamiltonian is locally equivalent to $H$ since they differ only by local terms (Rule 6). Furthermore, since

$$
\left(c H_{A}\right) \otimes\left(H_{B} / c\right)=H_{A} \otimes H_{B}
$$

we may assume $\lambda_{A}^{\max }-\lambda_{A}^{\min }=\lambda_{B}^{\max }-\lambda_{B}^{\min }=\Delta$ without loss of generality.

Having put all product Hamiltonians into a standard form, we are ready to show that they can reversibly simulate each other. By the transitivity of reversible simulation, it suffices to show that all product Hamiltonians can reversibly simulate the Ising interaction $H_{\text {Ising }}=\sigma_{z} \otimes \sigma_{z}$.

Lemma 2: Any tensor product Hamiltonian $H=H_{A} \otimes H_{B}$ can reversibly simulate the Ising interaction

$$
H \longleftrightarrow H_{\text {Ising }}, \quad \gamma_{H_{\text {Ising }} \mid H}=\frac{1}{4} \Delta^{2} .
$$

Proof: For any nonlocal $H_{1}$ and $H_{2}$, we have

$$
\gamma_{H_{1} \mid H_{2}} \gamma_{H_{2} \mid H_{1}} \leq 1 \text {. }
$$

Otherwise, we could use $H_{1}$ to simulate itself with simulation rate greater than 1 , which is a contradiction. ${ }^{1}$ It thus suffices to show that $\gamma_{H_{\text {Ising }} \mid H} \geq \Delta^{2} / 4$ and $\gamma_{H \mid H_{\text {Ising }}} \geq 4 / \Delta^{2}$.

Let $H$ act on a Hilbert space $\mathcal{H}_{A} \otimes \mathcal{H}_{B}$ with dimensions $d_{A}$ and $d_{B}$. Since $H=H_{A} \otimes H_{B}$ is in the standard form, we may write

$$
\begin{aligned}
H=\frac{1}{4} \Delta^{2} \operatorname{diag}\left(1, a_{2}, a_{3}, \ldots, a_{d_{A}-1},-1\right) & \\
& \otimes \operatorname{diag}\left(1, b_{2}, b_{3}, \ldots, b_{d_{B}-1},-1\right)
\end{aligned}
$$

where

$$
\begin{aligned}
& 1=a_{1} \geq a_{2} \geq \cdots \geq a_{d_{A}}=-1 \\
& 1=b_{1} \geq b_{2} \geq \cdots \geq b_{d_{B}}=-1
\end{aligned}
$$

and the corresponding eigenvectors are $|j\rangle_{A}\left(1 \leq j \leq d_{A}\right)$ and $|j\rangle_{B}\left(1 \leq j \leq d_{B}\right)$.

${ }^{1}$ If $\gamma_{H_{1} \mid H_{2}} \gamma_{H_{2} \mid H_{1}}>1$, then we could concatenate several simulations $H_{1} \longrightarrow H_{2} \longrightarrow H_{1} \longrightarrow \cdots \longrightarrow H_{2} \longrightarrow H_{1}$ to obtain that the optimal simulation rate $\gamma_{H_{1} \mid H_{1}}$ is infinite. Recalling that any bipartite nonlocal Hamiltonian can simulate any other one at finite rate, we would conclude that $\gamma_{H \mid H}$ is also infinite for any bipartite Hamiltonian. This would contradict, for instance, the results of [2] showing that $\gamma_{H \mid H}=1$ for all nonlocal two-qubit Hamiltonians.
We can simulate the Ising interaction using $H$ by restricting to the subspace spanned by the extremal eigenvectors of $H_{A}$ and $H_{B}\left(|1\rangle_{A}|1\rangle_{B},\left|d_{A}\right\rangle_{A}|1\rangle_{B},|1\rangle_{A}\left|d_{B}\right\rangle_{B},\left|d_{A}\right\rangle_{A}\left|d_{B}\right\rangle_{B}\right)$ according to Rule 8 . In this subspace, $H$ acts as $\left(\Delta^{2} / 4\right) H_{\text {Ising. }}$. Therefore, we have

$$
\gamma_{H_{\text {Ising }} \mid H} \geq \Delta^{2} / 4
$$

In order to show how to use the Ising interaction $H_{\text {Ising }}$ to simulate $H$, we consider a concatenation of two simulations

$$
H_{\text {Ising }} \longrightarrow H^{\prime \prime} \longrightarrow H \text {. }
$$

Here $H^{\prime \prime}=\frac{1}{4} \Delta^{2} H_{A A^{\prime}}^{\prime \prime} \otimes H_{B B^{\prime}}^{\prime \prime}$ acts on local Hilbert spaces of dimensions $2 d_{A}$ and $2 d_{B}$, and reads

$$
\begin{aligned}
H^{\prime \prime}=\frac{1}{4} \Delta^{2} \operatorname{diag}\left(1,-1, a_{2},-a_{2}, \ldots,-1,1\right) \\
\otimes \operatorname{diag}\left(1,-1, b_{2},-b_{2}, \ldots,-1,1\right) .
\end{aligned}
$$

Clearly, we can use Rule 8 to simulate $H$ by $H^{\prime \prime}$ with unit simulation rate. Therefore, we need only focus on the simulation of $H^{\prime \prime}$ by $H_{\text {Ising. }}$. In turn, this can be decomposed into two similar simulations

$$
H_{\text {Ising }} \longrightarrow H_{A A^{\prime}}^{\prime \prime} \otimes \sigma_{z} \longrightarrow H_{A A^{\prime}}^{\prime \prime} \otimes H_{B B^{\prime}}^{\prime \prime}
$$

each one with unit rate. In order to simulate $H_{A A^{\prime}}^{\prime \prime} \otimes \sigma_{z}$ using $\sigma_{z} \otimes \sigma_{z}$, we append a $d_{A}$-dimensional ancilla $A$ to qubit $A^{\prime}$

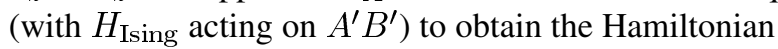

$$
\begin{aligned}
H_{\text {Ising }}^{\prime} & =\left(I \otimes \sigma_{z, A^{\prime}}\right) \otimes \sigma_{z} \\
& =\operatorname{diag}(1,-1,1,-1, \ldots, 1,-1) \otimes \sigma_{z} .
\end{aligned}
$$

We define

$$
p_{j}:=\left(a_{j}+1\right) / 2
$$

so that

$$
1=p_{1} \geq p_{2} \geq \cdots \geq p_{d_{A}}=0 .
$$

Furthermore, we define $d_{A}$ local unitary operations $\left\{U_{j}\right\}_{j=1}^{d_{A}}$, where $U_{j}$ exchanges the $(2 j-1)$ th and $(2 j)$ th basis vectors of $A A^{\prime}$. To evolve under $H_{A A^{\prime}}^{\prime \prime} \otimes \sigma_{z}$ for a small time $\delta$, we apply each $U_{j}$ at time $t=p_{j} \delta$ and $U_{j}^{\dagger}$ at time $t=\delta$. Equivalently, we can use Rule 7 with an appropriate probability distribution and set of unitaries. Thus, we can use $H_{\text {Ising }}$ to simulate $H_{A A^{\prime}}^{\prime \prime} \otimes \sigma_{z}$ with unit efficiency. The second simulation in (32) is achieved similarly. The overall rate for $H_{\text {Ising }}$ to simulate $H^{\prime \prime}$ or $H$ is thus $4 / \Delta^{2}$ by Rule 1 .

We have shown that any product Hamiltonian $H$ can reversibly simulate the Ising interaction $H_{\text {Ising }}$ with rate $\gamma_{H_{\text {Ising }} \mid H}=K_{\otimes}(H)$, where

$$
K_{\otimes}(H)=\frac{1}{4} \Delta_{A} \Delta_{B}
$$

Our main result then follows.

Theorem 3: Any product Hamiltonian $H$ can reversibly simulate any other product Hamiltonian $H^{\prime}$, with simulation rate given by

$$
\gamma_{H^{\prime} \mid H}=\frac{K_{\otimes}(H)}{K_{\otimes}\left(H^{\prime}\right)} .
$$


As discussed previously, in general a bipartite Hamiltonian $H$ cannot reversibly simulate $-H$. Similarly, in general, $H$ cannot reversibly simulate its complex conjugate $H^{*}$, nor the Hamiltonian $H^{\diamond}$ resulting from swapping systems $A$ and $B$. However, for product Hamiltonians, all these Hamiltonians are locally equivalent.

Corollary 4: For any product Hamiltonian $H$

$$
K_{\otimes}(H)=K_{\otimes}(-H)=K_{\otimes}\left(H^{*}\right)=K_{\otimes}\left(H^{\diamond}\right) .
$$

Theorem 3 can be extended to the case of a sum of bipartite product Hamiltonians acting on separate systems. If $H_{1}$ and $\mathrm{H}_{2}$ are two Hamiltonians acting, respectively, on bipartite systems $A_{1} B_{1}$ and $A_{2} B_{2}$, we let $H_{1} \boxplus H_{2}$ denote their sum. ${ }^{2}$ In fact, $H_{1} \boxplus H_{2}$ can reversibly simulate a product Hamiltonian $H$ acting on a single bipartite system $A B$.

Lemma 5: If $H_{1}, H_{2}$, and $H^{\prime}$ are product Hamiltonians, the simulation

$$
H_{1} \boxplus H_{2} \longleftrightarrow H^{\prime}
$$

can be achieved reversibly, with simulation rate

$$
\gamma_{H^{\prime} \mid H_{1} \boxplus H_{2}}=\gamma_{H^{\prime} \mid H_{1}}+\gamma_{H^{\prime} \mid H_{2}}
$$

Proof: Because of Theorem 3, we only need to show that the Hamiltonian $H^{\prime}=c H_{\text {Ising }} \boxplus d H_{\text {Ising }}, c, d \in \mathbb{R}$, can reversibly simulate $H=(|c|+|d|) H_{\text {Ising }}$ at unit rate. In addition, Corollary 4 implies that we need only consider the case $c, d>0$.

By Rule $2, H=(c+d) H_{\text {Ising }}$ is locally equivalent to

$$
J_{1}=(c+d) \sigma_{z A} \otimes \sigma_{z B} \otimes I_{A^{\prime} B^{\prime}} .
$$

In turn, using local unitaries to swap $A$ with $A^{\prime}$ and $B$ with $B^{\prime}$ (Rule 4$), J_{1}$ is locally equivalent to

$$
J_{2}=(c+d) I_{A B} \otimes \sigma_{z A^{\prime}} \otimes \sigma_{z B^{\prime}} .
$$

Then we can simulate $H^{\prime}=[c /(c+d)] J_{1}+[d /(c+d)] J_{2}$ by convex combination (Rule 5) of $J_{1}$ and $J_{2}$, which shows that $\gamma_{H^{\prime} \mid H} \geq 1$.

For the reverse simulation, note that $H^{\prime}$ is locally equivalent to each of the following four Hamiltonians:

$$
\begin{aligned}
& c\left(\sigma_{z A} \otimes I_{A^{\prime}} \otimes \sigma_{z B} \otimes I_{B^{\prime}}\right)+d\left(I_{A} \otimes \sigma_{z A^{\prime}} \otimes I_{B} \otimes \sigma_{z B^{\prime}}\right), \\
& c\left(I_{A} \otimes \sigma_{z A^{\prime}} \otimes \sigma_{z B} \otimes I_{B^{\prime}}\right)+d\left(\sigma_{z A} \otimes I_{A^{\prime}} \otimes I_{B} \otimes \sigma_{z B^{\prime}}\right), \\
& c\left(\sigma_{z A} \otimes I_{A^{\prime}} \otimes I_{B} \otimes \sigma_{z B^{\prime}}\right)+d\left(I_{A} \otimes \sigma_{z A^{\prime}} \otimes \sigma_{z B} \otimes I_{B^{\prime}}\right), \\
& c\left(I_{A} \otimes \sigma_{z A^{\prime}} \otimes I_{B} \otimes \sigma_{z B^{\prime}}\right)+d\left(\sigma_{z A} \otimes I_{A^{\prime}} \otimes \sigma_{z B} \otimes I_{B^{\prime}}\right) .
\end{aligned}
$$

Each of these Hamiltonians can be obtained from $H^{\prime}$ according to Rule 4 by swapping $A$ with $A^{\prime}$ and $B$ with $B^{\prime}$ as necessary. An equally weighted convex combination of these four Hamiltonians gives, after rearranging terms

$$
\frac{c+d}{4}\left(\sigma_{z A} \otimes I_{A^{\prime}}+I_{A} \otimes \sigma_{A^{\prime}}\right) \otimes\left(\sigma_{z B} \otimes I_{B^{\prime}}+I_{B} \otimes \sigma_{B^{\prime}}\right),
$$

a tensor product Hamiltonian with $\Delta^{2}=4(c+d)$. Therefore, $\gamma_{H \mid H^{\prime}} \geq 1$, which completes the proof.

${ }^{2}$ We use the symbol $\boxplus$ rather than + to emphasize that the Hamiltonians being summed act on different pairs of systems. In other words, $H_{A B} \boxplus H_{A^{\prime} B^{\prime}}=$ $H_{A B} \otimes I_{A^{\prime} B^{\prime}}+I_{A B} \otimes H_{A^{\prime} B^{\prime}}$.
It follows from Lemma 5 that $K_{\otimes}$ is additive under the sum of product Hamiltonians acting on different pairs of systems

$$
K_{\otimes}\left(H_{1} \boxplus H_{2}\right)=K_{\otimes}\left(H_{1}\right)+K_{\otimes}\left(H_{2}\right) .
$$

More generally, for $H=\boxplus_{i} H_{i}$ and $H^{\prime}=\boxplus_{i} H_{i}^{\prime}$, where all $H_{i}$ and $H_{i}^{\prime}$ are bipartite product Hamiltonians, we can perform the simulation

$$
H=\boxplus_{i} H_{i} \longleftrightarrow H^{\prime}=\bigoplus_{i} H_{i}^{\prime}
$$

reversibly, with simulation rate given by

$$
\gamma_{H^{\prime} \mid H}=\frac{\sum_{i} K_{\otimes}\left(H_{i}\right)}{\sum_{i} K_{\otimes}\left(H_{i}^{\prime}\right)} .
$$

Finally, in the Appendix, we present a case of a reversible Hamiltonian simulation that is possible when in addition to local operations and ancillas, catalytic pre-shared entanglement is available to Alice and Bob. The simulation can be made reversible only in the presence of entanglement, but the entanglement is not used up during the simulation [10].

\section{ENTANGLEMENT CAPACITY}

In this section, we use the results on reversible simulation from Section III to determine the optimal way a product Hamiltonian can be used to produce entanglement.

The problem of optimal entanglement generation by an interaction Hamiltonian has been approached in different ways. In [6], the single-shot scenario was considered. This corresponds to the setting in which an interaction $H$ is used, with the help of fast local unitary transformations (but without control over local ancillas), to maximize the rate of increase of entanglement between a single copy of the two interacting systems. This situation is of interest for many present-day experiments aiming to produce entangled states in quantum optics, nuclear magnetic resonance, or condensed matter systems [11]. The optimal rate at which entanglement can be generated in the single-shot scenario is known as the entanglement capability of $H, \Gamma_{H}$, and its value has been determined for any two-qubit Hamiltonian [6]. For the Ising interaction it reads

$$
\Gamma_{H_{\text {Ising }}}=\alpha \approx 1.9123
$$

where

$$
\alpha:=2 \max _{x \in[0,1]} \sqrt{x(1-x)} \log \left(\frac{x}{1-x}\right)
$$

with the maximum obtained at $x_{0} \approx 0.9168$.

In contrast, [7] considers entanglement generation in the asymptotic scenario, where many copies of the interacting systems (and local ancillas) can be used collectively to produce entanglement, possibly at a higher rate than in the single-shot case. The asymptotic entanglement capacity (or entanglement capacity for short) of interaction $H$, denoted $E_{H}$, is of interest in the context of understanding the ultimate limitations of quantum-mechanical systems to process quantum information. Bennett et al. [7] showed that entanglement capacities equal entanglement capabilities when ancillas are allowed in the single-shot setting. 
Finally, it was shown in [8] that for a number of two-qubit Hamiltonians, all single-shot and asymptotic scenarios lead to the same optimal entanglement generation rates. In particular

$$
E_{H_{\text {Ising }}}=\Gamma_{H_{\text {Ising }}}=\alpha \text {. }
$$

Combining (3) and Theorem 3, we obtain an expression for the entanglement capacity of any product Hamiltonian $H$, since we have $E_{H}=K_{\otimes}(H) E_{H_{\text {Ising }}}$.

Theorem 6: For any product Hamiltonian $H$

$$
E_{H}=\frac{\alpha}{4} \Delta_{A} \Delta_{B}
$$

Similarly, we can compute $E_{H_{1} \boxplus H_{2}}$ for the sum of two product Hamiltonians $H_{1}$ and $H_{2}$ acting on different pairs of systems

$$
E_{H_{1} \boxplus H_{2}}=E_{H_{1}}+E_{H_{2}} \text {. }
$$

In fact, (52) also corresponds to the single-shot capability $\Gamma_{H}$, since it can be obtained without using ancillas.

Corollary 7: For any product Hamiltonian $H$

$$
\Gamma_{H}=\frac{\alpha}{4} \Delta_{A} \Delta_{B}
$$

Proof: The explicit optimal input state is

$$
|\psi\rangle=\sqrt{x_{0}}|+\rangle_{A} \otimes|+\rangle_{B}+i \sqrt{1-x_{0}}|-\rangle_{A} \otimes|-\rangle_{B}
$$

where

$$
| \pm\rangle_{A}=\frac{1}{\sqrt{2}}\left(|1\rangle_{A} \pm\left|d_{A}\right\rangle_{A}\right)
$$

and, similarly, for system $B$. Here $|1\rangle_{A}$ and $\left|d_{A}\right\rangle_{A}$ represent the eigenstates of $H_{A}$ corresponding to the largest and smallest eigenvalues, respectively. That this state achieves $\Gamma_{H}$ can be seen by substitution into [8, eqs. (17) and (18)].

Likewise, (53) can also be achieved without ancillas, because the protocol used in Lemma 5 does not involve ancillas

$$
\Gamma_{H_{1} \boxplus H_{2}}=\Gamma_{H_{1}}+\Gamma_{H_{2}} \text {. }
$$

As a side remark, [12] has reported the restricted case of (52) in which $H_{A}$ and $H_{B}$ have eigenvalues \pm 1 , but this property has no special significance; only the tensor product structure is important.

Equation (52) could also be proved directly using essentially the same arguments that appear in [8]. Using the observation that the matrix with elements

$$
4\left|\left(H_{A}\right)_{j k}\left(H_{B}\right)_{j k}\right| / \Delta^{2}
$$

(where the matrix elements of $H_{A}$ and $H_{B}$ are taken in any orthonormal basis) is doubly substochastic, an upper bound on $E_{H}$ can be obtained just as in [8, eq. (26)]. However, the approach using reversible simulation is more powerful, since it applies to any capacity, even those for which the capacity of $H_{\text {Ising }}$ is yet to be found, such as the capacity for communicating classical information.

Finally, we note that Theorem 6 can be extended to Hamiltonians that can be reversibly simulated using catalytic entanglement, such as those mentioned in Section III and further described in the Appendix. This class of Hamiltonians includes, as a special case, the full set of two-qubit Hamiltonians of the form $\mu_{x} \sigma_{x} \otimes \sigma_{x}+\mu_{y} \sigma_{y} \otimes \sigma_{y}$ considered in [8]. In the context of asymptotic entanglement capacity, catalytic resources need not be considered as additional requirements since the cost of first obtaining any catalytic resource can be made negligible [7]. However, it turns out that for the Hamiltonians discussed in the Appendix, catalytic entanglement is actually not necessary to achieve the entanglement capacity.

\section{Strength Measures For Bipartite Hamiltonians}

In this section, we show that the notion of nonlocal Hamiltonian simulation can be used to define measures of the strength of a nonlocal Hamiltonian. We proceed along the lines of [9], which introduces a formalism of strength measures for nonlocal quantum operations, with the goal of quantifying their nonlocality. Three necessary axioms and a number of other desirable properties for such measures were proposed. Here we show that in the case of nonlocal Hamiltonians, a single axiom implies many of these properties.

We denote a strength measure for Hamiltonian $H$ as $K(H)$. The only requirement we impose on $K(H)$ is that it does not increase under Hamiltonian simulation by local manipulations. That is, if $H$ can be used to simulate $H^{\prime}$ at a rate $\gamma_{H^{\prime} \mid H}$, then the strength measure for $\gamma_{H^{\prime} \mid H} H^{\prime}$ should be no greater than that for $H$. This is motivated by the idea that any measure of the nonlocality of an interaction should not increase under local manipulations.

Axiom-Monotonicity: Any strength measure $K(H)$ must satisfy

$$
K(H) \geq \gamma_{H^{\prime} \mid H} K\left(H^{\prime}\right)
$$

for any two Hamiltonians $H, H^{\prime} .{ }^{3}$

In the following, we exclude the trivial strength measure $K(H)=0$ for all $H$. Likewise, we exclude any unphysical strength measure that is infinite for a bounded $H$.

We have already encountered two examples of nontrivial functions that do not increase under local manipulations: i) any capacity $C_{H}$ satisfies the Axiom because of (1), and ii) for any fixed target Hamiltonian $H_{\text {target }}$, the simulation rate $\gamma_{H_{\text {target }} \mid H}$ satisfies the Axiom as a function of $H$. In particular, the function

$$
K_{\otimes}(H):=\gamma_{H_{\text {Ising }} \mid H}
$$

used in Section IV is a strength measure.

A function $K(H)$ satisfying the Axiom automatically has a number of properties that we describe next for the bipartite case (although much of the discussion can be generalized to more

\footnotetext{
${ }^{3}$ One might also be interested in defining a strength measure in a setting where more than just a single Hamiltonian is available. Suppose we can use two switchable Hamiltonians $H_{1}$ and $H_{2}$ to achieve some task, and that we can only have one Hamiltonian switched on at a time. Let $p$ and $1-p(0 \leq p \leq 1)$ characterize the relative frequency with which we use each of the two Hamiltonians. Then we can define a function $K\left(p H_{1} ;(1-p) H_{2}\right)$ as a strength measure. More generally, $K$ may have any number of arguments. Suppose we can use $n$ Hamiltonians $\left\{p_{1} H_{1} ; p_{2} H_{2} ; \cdots ; p_{n} H_{n}\right\}$, where $p_{i}\left(p_{i} \geq 0, \sum_{i} p_{i}=1\right)$ indicates the relative frequency of $H_{i}$, to simulate Hamiltonians $\left\{q_{1} H_{1}^{\prime} ; q_{2} H_{2}^{\prime} ; \cdots ; q_{m} H_{m}^{\prime}\right\}$. Let $\gamma_{\left\{q_{i} H_{i}^{\prime}\right\} \mid\left\{p_{i} H_{i}\right\}}$ denote the rate at which this can be achieved. Then we would require that a strength measure $K$ fulfills $K\left(\left\{p_{i} H_{i}\right\}\right) \geq \gamma_{\left\{q_{i} H_{i}^{\prime}\right\} \mid\left\{p_{i} H_{i}\right\}} K\left(\left\{q_{i} H_{i}^{\prime}\right\}\right)$. Several additional properties can be derived for $K$. In particular, Rule 5 implies that $K\left(\left\{p_{i} H_{i}\right\}\right) \geq K\left(\sum_{i} p_{i} H_{i}\right)$.
} 
than two systems). Several of these properties appeared in the original formulation in [9].

Property 1-Positivity:

$$
K(H) \geq 0
$$

with equality if and only if $H$ is local.

Property 2-Homogeneity: For any $c \geq 0$

$$
K(c H)=c K(H) .
$$

Property 3-Stability Under Ancillas: For any ancillary system $A^{\prime} B^{\prime}$

$$
K(H)=K\left(H \otimes I_{A^{\prime} B^{\prime}}\right)
$$

Property 4-Local Unitary Invariance: For any local unitary operation $U=U_{A} \otimes U_{B}$

$$
K(H)=K\left(U H U^{\dagger}\right) .
$$

Property 5-Invariance Under Local Addition: For any local Hamiltonian $H_{0}$

$$
K(H)=K\left(H+H_{0}\right) .
$$

Property 6-Local Unitary Mixing: For any set of local unitary operators $U_{i}=U_{A, i} \otimes U_{B, i}$ and probability distribution $p_{i}$

$$
K(H) \geq K\left(\sum_{i} p_{i} U_{i} H U_{i}^{\dagger}\right) .
$$

Property 7-Reduction to a Local Subspace: Let $J_{\|}$denote the $m \times m$ upper left submatrix of $J$, and $K_{\|}$the $m^{\prime} \times m^{\prime}$ upper left submatrix of $K$. Let $H=\sum_{i} J_{i} \otimes K_{i}$ be a product expansion of a bipartite Hamiltonian $H$, and let $H^{\prime}=\sum_{i} J_{i \|} \otimes$ $K_{i \|}$ be its restriction to the upper left subspace. Then

$$
K(H) \geq K\left(H^{\prime}\right) .
$$

Property 8-Continuity: For any nonlocal Hamiltonian $H$ and any bounded Hermitian operator $J$

$$
\lim _{\epsilon \rightarrow 0} K(H+\epsilon J)-K(H)=0 .
$$

We now derive Properties 1-8 from the Axiom and our simulation rules in Section II. First we prove Property 1.

Proof: Let $H_{0}$ be any local Hamiltonian and $H$ be any bounded nonlocal Hamiltonian. $H_{0}$ cannot simulate $H$, so $\gamma_{H \mid H_{0}}=0$. The Axiom then implies $K\left(H_{0}\right) \geq 0$. Through local control, $H_{0}$ can be simulated without using $H$, so $\gamma_{H_{0} \mid H}>0$. The Axiom then implies $K(H) \geq 0$. However, $\gamma_{H_{0} \mid H}$ is unbounded. If $K(H)$ is bounded, $K\left(H_{0}\right)$ must be 0 . Finally, if $K(H)=0$, the fact that it can simulate all other (bounded) Hamiltonians $H^{\prime}$ with nonzero rate implies $K\left(H^{\prime}\right)=0$, which is excluded. Thus, $K(H)>0$.

Properties 2-7 follow from the simulations described, respectively, in Rules 1, 2, 4, and 6-8.

Finally, the proof of Property 8 is based on the fact that any two nonlocal bipartite Hamiltonians can simulate each other with nonzero rate.
Proof: Because nonlocal strength measures are invariant under addition of local terms (Property 5), we can focus on a Hamiltonian $H$ without local terms, that is, $\operatorname{tr}_{A} H=\operatorname{tr}_{B} H=0$, and similarly for $J$. Consider first the case where $J$ is proportional to $H$. Then Property 2 ensures continuity. Suppose now that $J$ is not proportional to $H$, so that $H+\epsilon J \neq 0 \forall \epsilon$ and is therefore always nonlocal. We then consider the following two simulations:

$$
\begin{aligned}
& H \longrightarrow H+\epsilon J \\
& H+\epsilon J \longrightarrow H .
\end{aligned}
$$

A possible procedure for (69) is to use $H$ to simulate itself at rate $\gamma_{H \mid H}$ and $\epsilon J$ at rate $\gamma_{\epsilon J \mid H}$, and to add the two Hamiltonians together by Rule 5 . This gives a lower bound on the optimal simulation rate

$$
\gamma_{H+\epsilon J \mid H} \geq\left(\frac{1}{\gamma_{H \mid H}}+\frac{1}{\gamma_{\epsilon J \mid H}}\right)^{-1}=\left(1+\frac{\epsilon}{\gamma_{J \mid H}}\right)^{-1} .
$$

Then, for small $\epsilon$

$$
\gamma_{H+\epsilon J \mid H} \geq 1-\frac{\epsilon}{\gamma_{J \mid H}}+O\left(\epsilon^{2}\right) .
$$

Similarly, for simulation (70) we arrive at

$$
\gamma_{H \mid H+\epsilon J} \geq 1-\frac{\epsilon}{\gamma_{-J \mid H+\epsilon J}}+O\left(\epsilon^{2}\right) .
$$

Furthermore, let

$$
g_{0}:=\min _{0 \leq \epsilon \leq \epsilon_{0}}\left(\gamma_{J \mid H}^{-1}, \gamma_{-J \mid H+\epsilon J}^{-1}\right)
$$

for any fixed $\epsilon_{0}>0$. Then the Axiom implies

$$
\begin{aligned}
K(H) & \geq\left(1-\epsilon g_{0}\right) K(H+\epsilon J)+O\left(\epsilon^{2}\right) \\
K(H+\epsilon J) & \geq\left(1-\epsilon g_{0}\right) K(H)+O\left(\epsilon^{2}\right)
\end{aligned}
$$

and

$$
|K(H)-K(H+\epsilon J)| \leq \epsilon g_{0} \bar{K}+O\left(\epsilon^{2}\right)
$$

where

$$
\bar{K}:=\max _{0 \leq \epsilon \leq \epsilon_{0}} K(H+\epsilon J)
$$

which proves the continuity of $K$.

For bipartite tensor product Hamiltonians, the following additional properties hold.

Property 9-Inverse Hamiltonian: For any product Hamiltonian $H=H_{A} \otimes H_{B}$

$$
K(-H)=K(H) .
$$

Property 10-Complex Conjugation: For any product Hamiltonian $H=H_{A} \otimes H_{B}$

$$
K\left(H^{*}\right)=K(H) .
$$

Property 11-Exchange: For any product Hamiltonian $H=$ $H_{A} \otimes H_{B}$ and for $H^{\diamond}$ the same Hamiltonian acting on exchanged systems

$$
K\left(H^{\diamond}\right)=K(H) .
$$


Property 12-Additivity: For product Hamiltonians $H_{1}$ and $H_{2}$ acting on different pairs of systems $A_{1} B_{1}$ and $A_{2} B_{2}$

$$
K\left(H_{1} \boxplus H_{2}\right)=K\left(H_{1}\right)+K\left(H_{2}\right) .
$$

Properties 9-11 follow from Corollary 4. Property 12 follows from Lemma 5.

The above Axiom relates the notion of strength measure to that of Hamiltonian simulation. The following lemma shows that identifying strength measures is of interest in order to establish bounds on simulation rates - and thereby also bounds on Hamiltonian capacities, as discussed in Section I.

Lemma 8: The optimal simulation rate $\gamma_{H^{\prime} \mid H}$ corresponds to an optimization over all possible strength measures $K$

$$
\gamma_{H^{\prime} \mid H}=\min _{K} \frac{K(H)}{K\left(H^{\prime}\right)} \text {. }
$$

Proof: From the Axiom, $\gamma_{H^{\prime} \mid H} \leq \frac{K(H)}{K\left(H^{\prime}\right)}$ for any strength measure $K$. In addition, the minimum is achieved by the strength measure $K(H)=\gamma_{H^{\prime} \mid H}$.

The many functions that fulfill the Axiom form a convex cone. In other words, if the functions $K_{1}$ and $K_{2}$ satisfy the Axiom, then so does $c_{1} K_{1}+c_{2} K_{2}$ for any $c_{1}, c_{2} \geq 0$. Of special interest is the subset of extremal strength measures, i.e., those that cannot be expressed as a positive sum of others, since we can restrict the optimization in (81) to such functions. Ideally, we would like to find a finite subset of extremal strength measures that form a complete set, in that optimization over this set gives the optimal rate $\gamma_{H^{\prime} \mid H}$ for all $H, H^{\prime}$. We will describe two examples of complete sets of extremal strength measures.

The first case corresponds to the simulation of two-qubit Hamiltonians by two-qubit Hamiltonians. Any such Hamiltonian can be written, up to local terms and local unitary transformations, as [6]

$$
H=\sum_{i=x, y, x} \lambda_{i} \sigma_{i} \otimes \sigma_{i}, \quad \lambda_{x} \geq \lambda_{y} \geq\left|\lambda_{z}\right| .
$$

The set of strength measures $K_{i}$

$$
\begin{aligned}
& K_{1}:=\lambda_{x} \\
& K_{2}:=\lambda_{x}+\lambda_{y}-\lambda_{z} \\
& K_{3}:=\lambda_{x}+\lambda_{y}+\lambda_{z}
\end{aligned}
$$

is a complete set of extremal strength measures in that any optimal rate $\gamma_{H^{\prime} \mid H}$ can be obtained from the optimization [2]

$$
\gamma_{H^{\prime} \mid H}=\min \left\{\frac{K_{1}(H)}{K_{1}\left(H^{\prime}\right)}, \frac{K_{2}(H)}{K_{2}\left(H^{\prime}\right)}, \frac{K_{3}(H)}{K_{3}\left(H^{\prime}\right)}\right\} .
$$

The second case corresponds to the simulation of product Hamiltonians by product Hamiltonians. We have seen that in this case the simulation can always be made reversible. This implies that, up to a multiplicative constant, there is a unique strength measure for product Hamiltonians

$$
K_{\otimes}(H):=\frac{1}{4} \Delta_{A} \Delta_{B}
$$

Indeed, because of reversibility, any function $K$ fulfilling the Axiom satisfies

$$
K(H)=\gamma_{H^{\prime} \mid H} K\left(H^{\prime}\right)
$$

for all product Hamiltonians $H, H^{\prime}$, so that

$$
\frac{K(H)}{K\left(H^{\prime}\right)}=\gamma_{H^{\prime} \mid H}=\frac{K_{\otimes}(H)}{K_{\otimes}\left(H^{\prime}\right)} .
$$

\section{DISCUSSION}

We have seen that all tensor product Hamiltonians can simulate each other reversibly, so that their nonlocal properties are characterized entirely by the quantity $K_{\otimes}(H)$ given in (6). This is an example of lossless interconversion of resources, an appealing situation in information theory. A related example is the problem of communication through a one-way classical channel. By Shannon's noisy coding theorem [13] together with the reverse Shannon theorem [14], all classical channels can simulate each other reversibly (in the presence of free shared randomness), and hence they can be characterized entirely in terms of a single quantity, their capacity. Similarly, in the presence of free shared entanglement, all one-way quantum channels can simulate each other reversibly (at least on certain input ensembles [15]), and thus they are characterized entirely in terms of their entanglement-assisted capacity for sending classical information.

In Section IV, we saw how Theorem 3 can be used to extend previous results for two-qubit Hamiltonians to product Hamiltonians. Another such extension can be obtained for the problem of using bipartite Hamiltonians to simulate bipartite unitary gates. In the case of two-qubit systems, it is known how to optimally produce any two-qubit gate using any two-qubit Hamiltonian [16]-[18]. Since all product Hamiltonians are equivalent to some multiple of the Ising interaction, this result immediately provides the optimal way to use any product Hamiltonian to simulate any two-qubit unitary gate, such as the controlled-not gate.

In view of our results, it will be interesting to improve our understanding of the properties of the Ising interaction. For example, as previously mentioned, a calculation of the communication capacity of the Ising interaction would provide a formula for the communication capacity of all product Hamiltonians.

It is also interesting to consider Hamiltonian simulation in the multipartite case. All of the Rules from Section II have multipartite analogues, and much of the general discussion on strength measures for Hamiltonians from Section V can be carried over as well. Furthermore, Theorem 3 can be generalized to more than two parties in the special case in which the individual tensor factors are traceless. However, much remains to be done in the general multipartite case.

Of course, the set of tensor product Hamiltonians is clearly a special subset of all bipartite Hamiltonians, and thus may not be representative of the general problem of bipartite Hamiltonian simulation. For example, we have seen that product Hamiltonians admit a total order, whereas even in the two-qubit case, general Hamiltonians only admit a partial order. Also, note that for product Hamiltonians, $H$ and $-H$ are locally equivalent, so that in particular, $E_{H}=E_{-H}$. However, while this is true for all two-qubit Hamiltonians, numerical evidence suggests that it is not true in general [19]. Understanding optimal Hamiltonian simulation and the capacities of Hamiltonians in the general case remains an interesting open problem. 


\section{APPENDIX}

Catalytic Simulation of a Sum of Two Product Hamiltonians With the SAME EXTREMAl EigENSPACE

As mentioned in Section III, Theorem 3 can be extended when catalytic entanglement is available. Consider a sum of two tensor product Hamiltonians

$$
H=J_{A} \otimes J_{B}+G_{A} \otimes G_{B}
$$

Let $J_{A}^{\mathrm{e}}$ denote the restriction of $J_{A}$ to the subspace corresponding to its two extremal eigenvalues. By Rule $3, J_{A}^{\mathrm{e}}$ can be assumed to be traceless. Let $G_{A}^{\mathrm{e}}, J_{B}^{\mathrm{e}}, G_{B}^{\mathrm{e}}$ be similarly defined. In terms of these Hamiltonians, we have the following.

Corollary 9: Given the resource of catalytic entanglement, $H=J_{A} \otimes J_{B}+G_{A} \otimes G_{B}$ is locally equivalent to $\left[\left(\Delta_{J}^{2}+\right.\right.$ $\left.\left.\Delta_{G}^{2}\right) / 4\right] H_{\text {Ising }}$ if the following conditions hold: i) $J_{A}^{\mathrm{e}}$ and $G_{A}^{\mathrm{e}}$ are supported on the same two-dimensional Hilbert space, and similarly for $J_{B}^{\mathrm{e}}$ and $G_{B}^{\mathrm{e}}$. ii) $\operatorname{tr} J_{A}^{\mathrm{e}} G_{A}^{\mathrm{e}}=\operatorname{tr} J_{B}^{\mathrm{e}} G_{B}^{\mathrm{e}}$.

Proof: $\left[\left(\Delta_{J}^{2}+\Delta_{G}^{2}\right) / 4\right] H_{\text {Ising }}$ can simulate $H$ termwise using Lemma 2 and Rule 5, with no need for catalytic entanglement.

The following procedure uses $H$ to simulate $\left[\left(\Delta_{J}^{2}+\Delta_{G}^{2}\right) / 4\right] H_{\text {Ising }}$.

1) Following Rule 8, Alice and Bob restrict to the extremal eigenspace, which is common to both terms in $H$ by condition i). This preserves the extremal eigenvalues. The resulting Hamiltonian is essentially a two-qubit Hamiltonian.

2) We can assume $J_{A}^{\mathrm{e}} \otimes J_{B}^{\mathrm{e}}=\left(\Delta_{J}^{2} / 4\right) \sigma_{z A} \otimes \sigma_{z B}$ by a local change of basis. This can be chosen so that $G_{A}^{\mathrm{e}} \otimes G_{B}^{\mathrm{e}}=$ $\left(\Delta_{G}^{2} / 4\right)\left(\cos \theta \sigma_{z A}+\sin \theta \sigma_{x A}\right) \otimes\left(\cos \theta \sigma_{z B}+\sin \theta \sigma_{x B}\right)$ for some $\theta$ because of condition ii).

3) A further local change of basis takes $J_{A}^{\mathrm{e}} \otimes J_{B}^{\mathrm{e}}+G_{A}^{\mathrm{e}} \otimes G_{B}^{\mathrm{e}}$ to its normal form $\left(\Delta_{x}^{2} / 4\right) \sigma_{x A} \otimes \sigma_{x B}+\left(\Delta_{z}^{2} / 4\right) \sigma_{z A} \otimes$ $\sigma_{z B}[6]$, where $\Delta_{x}^{2}+\Delta_{z}^{2}=\Delta_{J}^{2}+\Delta_{G}^{2}$.

4) Finally, $\left(\Delta_{x}^{2} / 4\right) \sigma_{x A} \otimes \sigma_{x B}+\left(\Delta_{z}^{2} / 4\right) \sigma_{z A} \otimes \sigma_{z B}$ can simulate $\left[\left(\Delta_{x}^{2}+\Delta_{z}^{2}\right) / 4\right] \sigma_{z A} \otimes \sigma_{z B}$ using catalytic entanglement [10].

This completes the proof.

This result allows us to calculate the entanglement capacities of the relevant Hamiltonians. If $H$ satisfies conditions i) and ii) of Corollary 9, then

$$
E_{H}=\frac{\alpha}{4}\left(\Delta_{J}^{2}+\Delta_{G}^{2}\right)
$$

There is an input state that achieves $E_{H}$ without making use of ancillas (and, in particular, without using catalytic entanglement), so $\Gamma_{H}=E_{H}$. As mentioned in Section IV, this generalizes the case $H=\mu_{x} \sigma_{x} \otimes \sigma_{x}+\mu_{y} \sigma_{y} \otimes \sigma_{y}$ considered in [8].

\section{REFERENCES}

[1] J. L. Dodd, M. A. Nielsen, M. J. Bremner, and R. Thew, "Universal quantum computation and simulation using any entangling Hamiltonian and local unitaries," Phys. Rev. A, vol. 65, p. 040301(R), 2002.

[2] C. H. Bennett, J. I. Cirac, M. S. Leifer, D. W. Leung, N. Linden, S. Popescu, and G. Vidal, "Optimal simulation of two-qubit Hamiltonians using general local operations," Phys. Rev. A, vol. 66, p. 012305, 2002.

[3] P. Wocjan, M. Rotteler, D. Janzing, and T. Beth, "Universal simulation of Hamiltonians using a finite set of control operations," Quant. Inform. Comp., vol. 2, pp. 133-150, 2002.

[4] M. A. Nielsen, M. J. Bremner, J. L. Dodd, A. M. Childs, and C. M. Dawson, "Universal simulation of Hamiltonian dynamics for quantum systems with finite-dimensional state spaces," Phys. Rev. A, vol. 66, p. $022317,2002$.

[5] G. Vidal and J. I. Cirac, "Optimal simulation of nonlocal Hamiltonians using local operations and classical communication," Phys. Rev. A, vol. 66, p. 022315, 2002.

[6] W. Dür, G. Vidal, J. I. Cirac, N. Linden, and S. Popescu, "Entanglement capabilities of nonlocal Hamiltonians," Phys. Rev. Lett., vol. 87, p. 137901, 2001.

[7] C. H. Bennett, A. W. Harrow, D. W. Leung, and J. A. Smolin, "On the capacities of bipartite Hamiltonians and unitary gates," IEEE Trans. Inform. Theory, vol. 49, pp. 1895-1911, Aug. 2003.

[8] A. M. Childs, D. W. Leung, F. Verstraete, and G. Vidal, "Asymptotic entanglement capacity of the Ising and anisotropic Heisenberg interactions," Quant. Inform. Comp., vol. 3, pp. 97-105, 2003.

[9] M. A. Nielsen, C. M. Dawson, J. L. Dodd, A. Gilchrist, D. Mortimer, T. J. Osborne, M. J. Bremner, A. W. Harrow, and A. Hines, "Quantum dynamics as a physical resource," Phys. Rev. A, vol. 67, p. 052301, 2003.

[10] G. Vidal and J. I. Cirac, "Catalysis in nonlocal quantum operations," Phys. Rev. Lett., vol. 88, p. 167903, 2002.

[11] Special Issue on Experimental Proposals for Quantum Computation, Fortschr. Phys., vol. 48, no. 9-11, 2000.

[12] X. Wang and B. C. Sanders, "Entanglement capability of self-inverse Hamiltonian evolution," Phys. Rev. A, vol. 68, p. 014301, 2003.

[13] C. E. Shannon, "A mathematical theory of communication," Bell Syst. Tech. J., vol. 27, pp. 379-423, 623-656, 1948.

[14] C. H. Bennett, P. W. Shor, J. A. Smolin, and A. V. Thapliyal, "Entanglement-assisted capacity of a quantum channel and the reverse Shannon theorem," IEEE Trans. Inform. Theory, vol. 48, pp. 2637-2655, Oct. 2002.

[15] C. H. Bennett, I. Devetak, P. W. Shor, and A. Winter, in preparation.

[16] G. Vidal, K. Hammerer, and J. I. Cirac, "Interaction cost of nonlocal gates," Phys. Rev. Lett., vol. 88, p. 237902, 2002.

[17] K. Hammerer, G. Vidal, and J. I. Cirac, "Characterization of nonlocal gates," Phys. Rev. A, vol. 66, p. 062321, 2002.

[18] A. M. Childs, H. L. Haselgrove, and M. A. Nielsen, "Lower bounds on the complexity of simulating quantum gates," Phys. Rev. A, vol. 68, p. $052311,2003$.

[19] A. M. Childs, D. W. Leung, and J. A. Smolin, unpublished. 